\title{
Simulation study on the effects of different flow conditions on the combustion of square fire
}

\author{
Tianxin $\mathrm{Li}^{1}$ and Yan $\mathrm{Jiao}^{1,2^{*}}$ \\ ${ }^{1}$ School of Environment and Energy Engineering, Anhui Jianzhu University, Hefei 230601, Anhui, China \\ ${ }^{2}$ Key Laboratory of Huizhou Architecture in Anhui Province, Anhui Jianzhu University, Hefei 230601, Anhui, China
}

\begin{abstract}
Combustion is a process in which light and heat are produced when a series of complex chemical reactions take place. Our life has long been inseparable from combustion, such as coal for power generation, fuel oil for car engines, rocket propulsion, waste incineration; but at the same time, with the development of the city, the damage caused by frequent fires is more serious. The purpose of this topic is to study the laws of plane flame combustion under different conditions. It is proposed to simulate the real plane fire combustion conditions with different flow conditions such as wind speed, combustion space and opening conditions, and propose relevant cognition and Countermeasures Based on this. Through the analysis of the above points, we can simulate the combustion situation of different weather and floors under different real conditions, different scale indoor environment and boundary conditions, so as to carry out new building requirements and planning, and lay a referential foundation for the evaluation and control of the fire scale.
\end{abstract}

\section{Introduction}

Combustion is a kind of chemical reaction that occurs when a combustible is mixed with oxygen under certain conditions. This reaction process will convert the specific chemical energy of the combustible into heat energy, which is used by human beings for functional conversion. Fire dynamics simulator (FDS) has been applied to simulate square fires [1].

Now with the progress of science and technology, we can use a relatively efficient and large-scale mechanical system to convert the heat generated by combustion into the mechanical energy we need, directly use the heat released by it. On the other hand, combustion will also bring devastating disasters to human beings at some times. We need to have a more substantive understanding of the phenomenon of combustion, and master its laws through a variety of research and practice. Whether it is disaster prevention or combustion utilization, the research on this aspect is of great significance. the comparison results are discussed.

\section{Methods and Model}

\subsection{Research method}

Pool fire simulation needs certain site materials, and with certain risks, because of the limitations of re-search scale and research site, the main combustion phenomenon in this paper will be studied by computer data simulation. It is proposed to use pyrosim and its own FDS fire simulation function for simulation study. The main process is divided into modeling, model processing, condition setting, measurement and positioning, image simulation and data processing. Through data simulation, the real fire phenomenon is simulated, statistical data, summarized rules and put forward relevant suggestions [2]-[4].

\subsection{Model establishment}

\subsubsection{Pyrosim and FDS}

The built-in modeling function of pyrosim is limited, and it is difficult to define and model the more complex geometry. Therefore, part of the solid modeling adopts SolidWorks modeling, and then exports and imports it into pyrosim module through STL format (Fig. 1a). When importing STL file, pay attention to unit conversion, and the STL file modeled in SolidWorks should pay attention to its initial coordinate position.

In FDS simulation, there are mainly the following steps: Edit Mesh, Edit Reaction, Edit Material, Edit Surface, Edit Vent, set Device, Set Output, Run SmokeView, Plot.

\subsubsection{Process for model}

The finite space combustion simulation is used here, so the standard cube combustion chamber is established. In order to ensure the accuracy of the experimental progress, the grid distribution of $x=30, y=30, z=45$ is adopted

\footnotetext{
* Corresponding author: jiao0418@mail.ustc.edu.cn
} 
in the standardized model (Figlb), which satisfies the Poisson distribution method in the Fourier transform formula (FFT), and it is not easy to cut off the grid at the boundary in the regular body.

After defining the mesh, define the boundary conditions in the surface menu. The inert and open used in the simulation are the boundary conditions of the system. The burner option is adopted for the newly built combustion window, and the default is adopted for the color and culture. When the standardized model is established, all parameters are tentatively defaulted.

Create a new vent and name buttom-vent1. Select the newly established fire as the surface attribute. Select $\mathrm{z}$ plane as the datum in the geometric attribute, coordinate 0.0 , boundary size $\min x=-0.25 \mathrm{~m}, \operatorname{Max} \mathrm{x}=0.25 \mathrm{~m}$, min $\mathrm{y}=-0.25 \mathrm{~m}, \operatorname{Max} \mathrm{y}=0.25 \mathrm{~m}$, and establish an independent combustion surface of $0.5 \mathrm{~m} * 0.5 \mathrm{~m}$.

Then we need to measure and collect the measured data. Here we use pyrosim's built-in device and detector to test the relevant data. First, we set ten thermocouples for measuring the vertical temperature change curve, and take the data in the following table respectively (Table 1):

Table 1. Space coordinate of thermocouples.

\begin{tabular}{lcccccccccc}
\hline No. 1 & 2 & 3 & 4 & 5 & 6 & 7 & 8 & 9 & 10 \\
\hline $\mathrm{X}$ & 0 & 0 & 0 & 0 & 0 & 0 & -0.25 & 0.25 & -0.25 & 2.5 \\
$\mathrm{Y}$ & 0 & 0 & 0 & 0 & 0 & 0 & 0 & 0 & 0 & 0 \\
$\mathrm{Z}$ & 0.5 & 1.0 & 1.5 & 2.0 & 2.5 & 3.0 & 1.0 & 1.0 & 1.5 & 1.5 \\
\hline
\end{tabular}

Set a section with the original base point as the center, $\mathrm{Y}$-axis plane and transverse direction, and set the following measurement indicators:

- Temperature

- Pressure

- Density

- Mix fraction

- Heat release rate per unit volume

- Velocity.

Table 2. Vent distribution characteristics

\begin{tabular}{|c|c|c|c|c|c|}
\hline Plane X & Min $Y$ & $\operatorname{Max} Y$ & $\operatorname{Min} Z$ & $\operatorname{Max} Z$ & Area \\
\hline Middle Small & -0.5 & 0.5 & 1.0 & 2.0 & 1 \\
\hline Top Small & -0.5 & 0.5 & 2.0 & 3.0 & 1 \\
\hline Bottom Small & -0.5 & 0.5 & 0 & 1.0 & 1 \\
\hline Middle Sig & -1.0 & 1.0 & 0.5 & 2.5 & 4 \\
\hline Open & All & All & All & All & All \\
\hline
\end{tabular}

\subsection{Parameter selection}

There are three groups of parameters in this experiment, which are: vent distribution characteris-tics (Table-2); vent geometry characteristics (Table-3); burner geometry characteristics (Table-4).

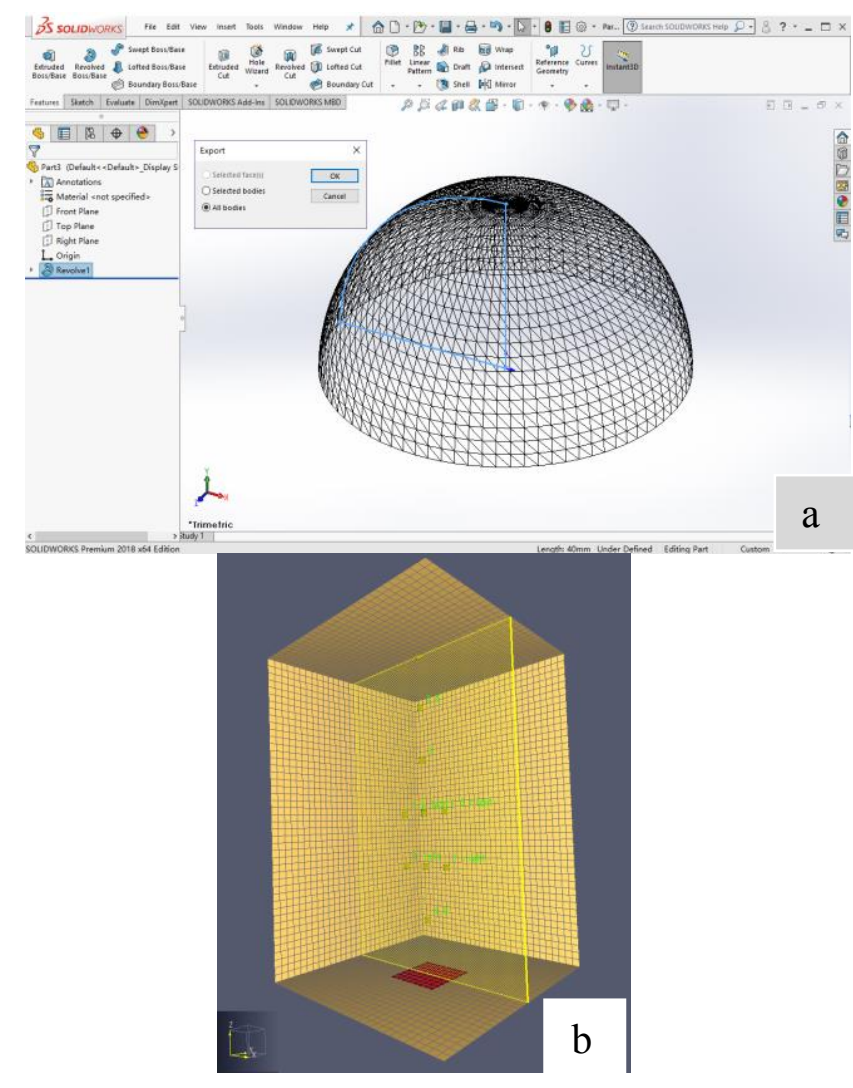

Figure 1. Complex model building and Establishment of combustion model

Table 3. Vent geometry characteristics

\begin{tabular}{ccccc}
\hline & Area & Speed & Altitude & Layout \\
\hline Wind Vent 1 & $1 * 1$ & 1 & Bottom & Single \\
\hline Wind Vent 2 & $1.4 * 1.4$ & 1 & Bottom & Single \\
\hline Wind Vent 3 & $1 * 1$ & 2 & Bottom & Single \\
\hline Wind Vent 4 & $1 * 1$ & 1 & Middle & Single \\
\hline Wind Vent 5 & $0.5 * 0.5 * 4$ & 1 & Vertical & Group \\
\hline
\end{tabular}

Table 4. Burner geometry characteristics

\begin{tabular}{lclllll}
\hline \multicolumn{5}{l}{ Area } & Shape & \multicolumn{3}{c}{ Layout } & \multicolumn{4}{l}{ Geometry HHRPU HHR } \\
\hline 1 & $1^{*} 1$ & Square & Single & Plane & 500 & 500 \\
\hline 2 & $1.4^{*} 1.4$ & Square & Single & Plane & 250 & 500 \\
\hline 3 & $1^{*} 1$ & Square & Single & Plane & 1000 & 1000 \\
\hline 4 & $0.5 * 0.5$ & Square*4 & Group & Matrix & 500 & 500 \\
\hline 5 & $0.5 * 0.5$ & Square*4 & Group & Row & 500 & 500 \\
\hline 6 & $\mathrm{R}=0.565$ & Square & Circular & Plane & 500 & 500 \\
\hline
\end{tabular}

\section{Results and Discussions}

\subsection{The effect of the opening characteristics}

As known, the opening scale and position are important factor for the combustion of the diffusion flame. In this paper, different opening characteristics are selected for comparison. There are middle small opening, top small opening, bottom small opening, middle large opening and full open type (as Table 2). 

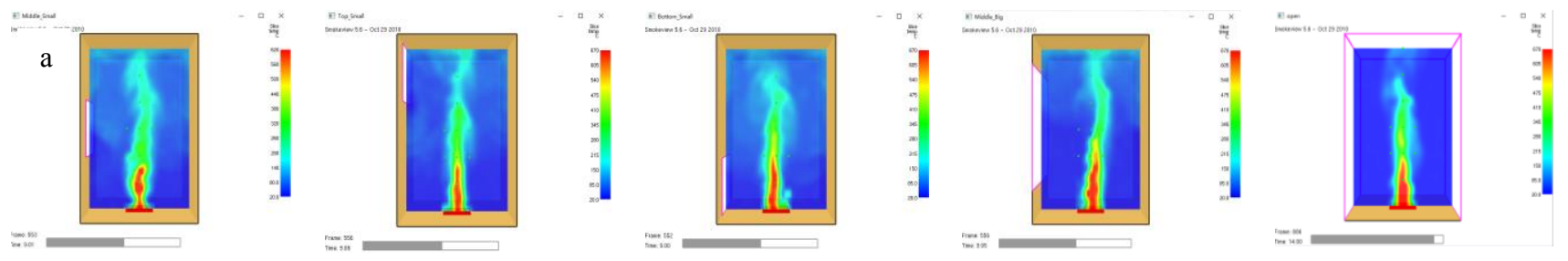

Figure 3.1 Flame combustion with different opening
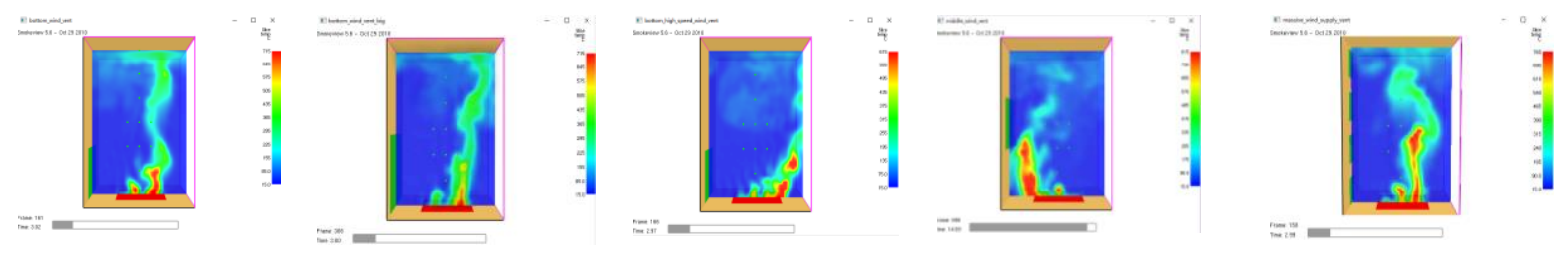

Figure 3.2 Flame combustion with different boundary

Physically, the fire burning is dominated by the radiative, convective and conductive heat feedback [5]. As Fig.3.1 showed, the combustion of the diffusion flame turned to be best effectively at the middle large opening than that of the small and the all opening; even though there is a largest space for the case of all opening. This may be caused by the more convectional heat loss.

\subsection{The effect of wind speed}

By studying the influence of different air inlet parameters on flame combustion, the simulation of boundary conditions in different environments is realized. There are the following experiments respectively:

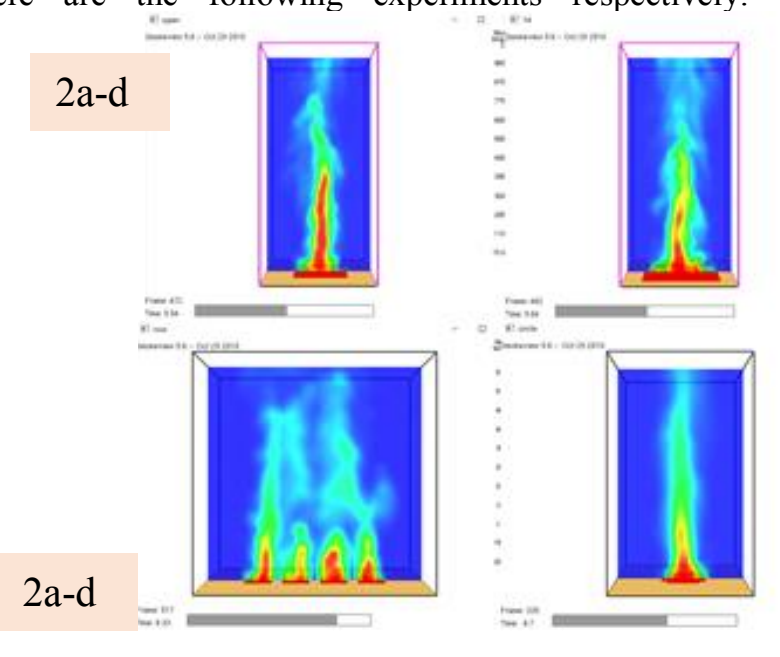

bottom standard air supply, bottom gale air supply, bottom high-speed air supply, middle standard air supply and decentralized air supply (Fig. 3.2).

It is found that the flame tilted more obviously in the direction of the wind in bottle opening with the increase of the wind speed (Fig. 3.2a-c), while the effect of the size of opening is not quite. However, the flame body turned toward the wall where the opening in the middle position comparing to the case with four vertical smaller opening which the total area is the same. It is probably due to the formation of a negative pressure that causes the flame to tilt inward, even though there is absolutely free space opposite of the large opening side.

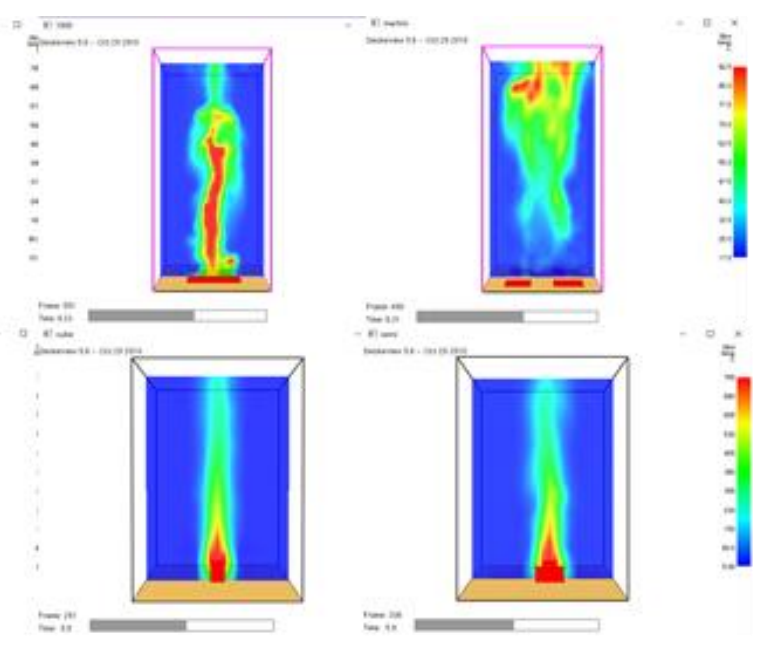

Figure 3.3 Flame combustion with different conditions

\subsection{The effect of burner and HRR}

Flame height from a diffusion fire source in a free boundary condition is corrected with the dimensionless heat release rate $\mathrm{Q}^{*}$, which depends on the geometry of the fire source and the potential intensity of the heat release rate [6]-[8].

According to the influence of different burners on combustion and flame characteristics, the following experiments are carried out: standard square, standard large square, standard square high power, matrix standard, array standard heat release, circular standard, cube standard, hemispherical standard.

Firstly, from the flame shape, there are some It could be found that the surface of flame body with square burner is much smoother than that of circle burner. This agrees well with the experimental theory and phenomena. Based on different shape of the burner, the four corners of the square burner would make small vortexes beside the bottom of the fire, which cause the turbulent flow pulsation (Fig. 3.3 1a-2b) [9]. Both of the heights of the 
two flames are nearly equal from the pictures by the observation.

Secondly, Dimensionless flame height is relation with the dimensionless heat release rate $Q^{*}$ [10]. It decreases the height of the fire with half standard heat release, while increases that of the fire with two times standard. It could be verified from the equations (1)-(2).

$$
\begin{gathered}
L_{f}{ }^{*}=\frac{L_{f}}{D} \\
Q^{*}=\frac{\dot{Q}}{\rho_{\infty} c_{p} T_{\infty} \sqrt{\mathrm{g} D D^{2}}}
\end{gathered}
$$

where $\rho_{\infty}, \quad c_{p}$ and $T_{\infty}$ are density, specific heat, and temperature of ambient air, respectively, and $\mathrm{g}$ is gravitational acceleration.

Thirdly, it is much more violently for the fire combustion of matrix arrangement with a little space between the fires (Fig.3.1d) than of that in large square (Fig.3.1c) [11].

\section{Conclusions}

This paper presented the numerical results on flame from multiple fire sources. According to the temperature distribution in the gas velocity distribution, when the flame burns in the lateral opening space, the flame will not be greatly affected. With the combustion going on, the whole flame will shift to the opposite direction of the opening side, while the size and position of the opening have complex influence on the whole system. In addition, the dispersed distribution of the burners is conducive to the combustion of the flame.

1. The FDS correctly predicts the flame height in simulating the flame sources through theorical equations.

2 . With the same heat release rate totally, more fire sources lead to higher flame height than a fire with a single large area as a result of air entrainment restriction.

3 . High heat release rate will bring more intense combustion phenomenon.

There will be more complex combustion phenomena in the distributed burner. For example, the matrix dispersed burner will significantly improve the flame height, and the array distribution will have a more complex influence law of its spacing. It comes to be the next work.

\section{Acknowledgements}

The authors gratefully acknowledge financial support from the Doctor Program of Anhui Jianzhu University (No.2018QD57), the Science and Technology Major projects of Anhui Province (18030801106, 16030801118), and the Key Research and Development Plan of Anhui Province, Granted (201904a07020070).

\section{References}

1. J.X. Wen, K. Kang, T. Donchev, J.M. Karwatzki. Fire Safety J. 42 (2), 127-138 (2007)
2. Hendrik Forkel, Johannes Janicka. Flow Turbu. Combust. 65 163-171 (2000).

3. A.F. Ghoniem, I. Lakkis, M. Steriou Proc. Combust. Inst. 26 1531-1539 (1996)

4. C.H. Wang, T. Lin, N. Li, H.P. Zheng. Renew. Energy. 96, 960-965 (2016)

5. J.G. Quintiere, Fundamentals of Fire Phenomena, John Wiley \& Sons Ltd, Chichester, UK, (2006)

6. A.A. Putnam, C.F. Speich, Proc. Combust. Inst. 9 (1) 867-877 (1963)

7. H.X. Wan, J. Ji, K.Y. Li, X.Y. Huang, J.H Sun, Y.M. Zhang. Proc. Combust. Inst. 36 3003-3010 (2017)

8. K. Wang, C.F. Tao, Q. Liu, Y.J. Qian, and P.X. He Exp. Heat Transf. 31(1) 22-31 (2018)

9. Y. Jiao, W. Gao, N.A. Liu, J. Lei, X.D. Xie, L.H. Zhang, R. Tu. Proc. Combust. Inst. 37 3967-3974 (2019).

10. W.G Weng, D. Kamikawa, Y. Fukuda, Y. Hasemi, K. Kagiya. Combust. Sci. and Technol. 176(12) 2105-2123 (2004)

11. D.T. Gottuk, B.Y. Lattimer, SFPE Handbook of Fire Protection Engineering, Springer (pp. 486-528 2016) 